\title{
Essential dimension of the spin groups in characteristic 2
}

\author{
Burt Totaro
}

The essential dimension of an algebraic group $G$ is a measure of the number of parameters needed to describe all $G$-torsors over all fields. A major achievement of the subject was the calculation of the essential dimension of the spin groups over a field of characteristic not 2, started by Brosnan, Reichstein, and Vistoli, and completed by Chernousov, Merkurjev, Garibaldi, and Guralnick [3, 4, 7], [17, Theorem 9.1].

In this paper, we determine the essential dimension of the spin group $\operatorname{Spin}(n)$ for $n \geq 15$ over an arbitrary field (Theorem 2.1). We find that the answer is the same in all characteristics. In contrast, for the groups $O(n)$ and $S O(n)$, the essential dimension is smaller in characteristic 2, by Babic and Chernousov [1].

In characteristic not 2 , the computation of essential dimension can be phrased to use a natural finite subgroup of $\operatorname{Spin}(2 r+1)$, namely an extraspecial 2-group, a central extension of $(\mathbf{Z} / 2)^{2 r}$ by $\mathbf{Z} / 2$. A distinctive feature of the argument in characteristic 2 is that the analogous subgroup is a finite group scheme, a central extension of $(\mathbf{Z} / 2)^{r} \times\left(\mu_{2}\right)^{r}$ by $\mu_{2}$, where $\mu_{2}$ is the group scheme of square roots of unity.

In characteristic not 2, Rost and Garibaldi computed the essential dimension of $\operatorname{Spin}(n)$ for $n \leq 14$ [6, Table 23B], where case-by-case arguments seem to be needed. We show in Theorem 3.1 that for $n \leq 10$, the essential dimension of $\operatorname{Spin}(n)$ is the same in characteristic 2 as in characteristic not 2 . It would be interesting to compute the essential dimension of $\operatorname{Spin}(n)$ in the remaining cases, $11 \leq n \leq 14$ in characteristic 2 .

This work was supported by NSF grant DMS-1303105. Thanks to Skip Garibaldi and Alexander Merkurjev for their suggestions. Garibaldi spotted a mistake in my previous description of the finite group scheme in the proof of Theorem 2.1.

\section{Essential dimension}

Let $G$ be an affine group scheme of finite type over a field $k$. Write $H^{1}(k, G)$ for the set of isomorphism classes of $G$-torsors over $k$ in the fppf topology. For $G$ smooth over $k$, this is also the set of isomorphism classes of $G$-torsors over $k$ in the etale topology.

Following Reichstein, the essential dimension $\operatorname{ed}(G)$ is the smallest natural number $r$ such that for every $G$-torsor $\xi$ over an extension field $E$ of $k$, there is a subfield $k \subset F \subset E$ such that $\xi$ is isomorphic to some $G$-torsor over $F$ extended to $E$, and $F$ has transcendence degree at most $r$ over $k$. (It is essential that $E$ is allowed to be any extension field of $k$, not just an algebraic extension field.) There are several survey articles on essential dimension, including [18, 16]. 
For example, let $q_{0}$ be a quadratic form of dimension $n$ over a field $k$ of characteristic not 2. Then $O\left(q_{0}\right)$-torsors can be identified with quadratic forms of dimension $n$, up to isomorphism. (For convenience, we sometimes write $O(n)$ for $O\left(q_{0}\right)$.) Thus the essential dimension of $O(n)$ measures the number of parameters needed to describe all quadratic forms of dimension $n$. Indeed, every quadratic form of dimension $n$ over a field of characteristic not 2 is isomorphic to a diagonal form $\left\langle a_{1}, \ldots, a_{n}\right\rangle$. It follows that the orthogonal group $O(n)$ in characteristic not 2 has essential dimension at most $n$; in fact, $O(n)$ has essential dimension equal to $n$, by one of the first computations of essential dimension [18, Example 2.5]. Reichstein also showed that the connected group $S O(n)$ in characteristic not 2 has essential dimension $n-1$ for $n \geq 3$ [18, Corollary 3.6].

For another example, for a positive integer $n$ and any field $k$, the group scheme $\mu_{n}$ of $n$th roots of unity is smooth over $k$ if and only if $n$ is invertible in $k$. Independent of that, $H^{1}\left(k, \mu_{n}\right)$ is always isomorphic to $k^{*} /\left(k^{*}\right)^{n}$. From that description, it is immediate that $\mu_{n}$ has essential dimension at most 1 over $k$. It is not hard to check that the essential dimension is in fact equal to 1.

One simple bound is that for any generically free representation $V$ of a group scheme $G$ over $k$ (meaning that $G$ acts freely on a nonempty open subset of $V$ ), the essential dimension of $G$ is at most $\operatorname{dim}(V)-\operatorname{dim}(G)$ [17, Proposition 5.1]. It follows, for example, that the essential dimension of any affine group scheme of finite type over $k$ is finite.

For a prime number $p$, the $p$-essential dimension $\operatorname{ed}_{p}(G)$ is a simplified invariant, defined by "ignoring field extensions of degree prime to $p$ ". In more detail, for a $G$-torsor $\xi$ over an extension field $E$ of $k$, define the $p$-essential dimension $\operatorname{ed}_{p}(\xi)$ to be the smallest number $r$ such that there is a finite extension $E^{\prime} / E$ of degree prime to $p$ such that $\xi$ over $E^{\prime}$ comes from a $G$-torsor over a subfield $k \subset F \subset E^{\prime}$ of transcendence degree at most $r$ over $k$. Then the $p$-essential dimension $\operatorname{ed}_{p}(G)$ is defined to be the supremum of the $p$-essential dimensions of all $G$-torsors over all extension fields of $k$.

The spin group $\operatorname{Spin}(n)$ is the simply connected double cover of $S O(n)$. It was a surprise when Brosnan, Reichstein, and Vistoli showed that the essential dimension of $\operatorname{Spin}(n)$ over a field $k$ of characteristic not 2 is exponentially large, asymptotic to $2^{n / 2}$ as $n$ goes to infinity [3]. As an application, they showed that the number of "parameters" needed to describe all quadratic forms of dimension $2 r$ in $I^{3}$ over all fields is asymptotic to $2^{r}$.

We now turn to quadratic forms over a field which may have characteristic 2 . Define a quadratic form $(q, V)$ over a field $k$ to be nondegenerate if the radical $V^{\perp}$ of the associated bilinear form is 0 , and nonsingular if $V^{\perp}$ has dimension at most 1 and $q$ is nonzero on any nonzero element of $V^{\perp}$. (In characteristic not 2, nonsingular and nondegenerate are the same.) The orthogonal group is defined as the automorphism group scheme of a nonsingular quadratic form [12, section VI.23]. For example, over a field $k$ of characteristic 2, the quadratic form

$$
x_{1} x_{2}+x_{3} x_{4}+\cdots+x_{2 r-1} x_{2 r}
$$

is nonsingular of even dimension $2 r$, while the form

$$
x_{1} x_{2}+x_{3} x_{4}+\cdots+x_{2 r-1} x_{2 r}+x_{2 r+1}^{2}
$$


is nonsingular of odd dimension $2 r+1$, with $V^{\perp}$ of dimension 1 . The split orthogonal group over $k$ is the automorphism group of one of these particular quadratic forms.

Babic and Chernousov computed the essential dimension of $O(n)$ and the smooth connected subgroup $O^{+}(n)$ over an infinite field $k$ of characteristic 2 [1]. (We also write $S O(n)$ for $O^{+}(n)$ by analogy with the case of characteristic not 2, even though the whole group $O(2 r)$ is contained in $S L(2 r)$ in characteristic 2.) The answer is smaller than in characteristic not 2. Namely, $O(2 r)$ has essential dimension $r+1$ (not $2 r$ ) over $k$. Also, $O^{+}(2 r)$ has essential dimension $r+1$ for $r$ even, and either $r$ or $r+1$ for $r$ odd, not $2 r-1$. Finally, the group scheme $O(2 r+1)$ has essential dimension $r+2$ over $k$, and $O^{+}(2 r+1)$ has essential dimension $r+1$. The lower bounds here are difficult, while the upper bounds are straightforward. For example, to show that $O(2 r)$ has essential dimension at most $r+1$ in characteristic 2 , write any quadratic form of dimension $2 r$ as a direct sum of 2-dimensional forms, thus reducing the structure group to $(\mathbf{Z} / 2)^{r} \times\left(\mu_{2}\right)^{r}$, and then use that the group $(\mathbf{Z} / 2)^{r}$ has essential dimension only 1 over an infinite field of characteristic 2 [1, proof of Proposition 13.1].

In this paper, we determine the essential dimension of $\operatorname{Spin}(n)$ in characteristic 2 for $n \leq 10$ or $n \geq 15$. Surprisingly, in view of what happens for $O(n)$ and $O^{+}(n)$, the results for spin groups are the same in characteristic 2 as in characteristic not 2 . For $n \leq 10$, the lower bound for the essential dimension is proved by constructing suitable cohomological invariants. It is not known whether a similar approach is possible for $n \geq 15$, either in characteristic 2 or in characteristic not 2 .

\section{Main result}

Theorem 2.1. Let $k$ be a field. For every integer $n$ at least 15, the essential dimension of the split group $\operatorname{Spin}(n)$ over $k$ is given by:

$$
\operatorname{ed}_{2}(\operatorname{Spin}(n))=\operatorname{ed}(\operatorname{Spin}(n))= \begin{cases}2^{n-1}-n(n-1) / 2 & \text { if } n \text { is odd } \\ 2^{(n-2) / 2}-n(n-1) / 2 & \text { if } n \equiv 2 \quad(\bmod 4) \\ 2^{(n-2) / 2}+2^{m}-n(n-1) / 2 & \text { if } n \equiv 0 \quad(\bmod 4)\end{cases}
$$

where $2^{m}$ is the largest power of 2 dividing $n$.

Proof. For $k$ of characteristic 0, this was proved by Chernousov and Merkurjev, sharpening the results of Brosnan, Reichstein, and Vistoli [4, Theorem 2.2]. Their argument works in any characteristic not 2, using the results of Garibaldi and Guralnick for the upper bounds [7. Namely, Garibaldi and Guralnick showed that for any field $k$ and any $n$ at least $15, \operatorname{Spin}(n)$ acts generically freely on the spin representation for $n$ odd, on each of the two half-spin representations if $n \equiv 2$ $(\bmod 4)$, and on the direct sum of a half-spin representation and the standard representation if $n \equiv 0(\bmod 4)$. Moreover, for $n$ at least 20 with $n \equiv 0(\bmod 4)$, $\operatorname{HSpin}(n)=\operatorname{Spin}(n) / \mu_{2}$ (the quotient different from $\left.O^{+}(n)\right)$ acts generically freely on a half-spin representation [7, Theorem 1.1].

It remains to consider a field $k$ of characteristic 2. Garibaldi and Guralnick's result gives the desired upper bound in most cases. Namely, for $n$ odd and at least 15 , the spin representation has dimension $2^{(n-1) / 2}$, and so ed( $\left.\operatorname{Spin}(n)\right) \leq$ $2^{(n-1) / 2}-\operatorname{dim}(\operatorname{Spin}(n))=2^{(n-1) / 2}-n(n-1) / 2$. For $n \equiv 2(\bmod 4)$, the half-spin 
representations have dimension $2^{(n-2) / 2}$, and so ed $(\operatorname{Spin}(n)) \leq 2^{(n-2) / 2}-n(n-1) / 2$. For $n=16$, since the spin group acts generically freely on the direct sum of a halfspin representation and the standard representation, $\operatorname{ed}(\operatorname{Spin}(n)) \leq 2^{(n-2) / 2}+n-$ $n(n-1) / 2(=24)$.

For $n$ at least 20 and divisible by 4, the optimal upper bound requires more effort. The following argument is modeled on Chernousov and Merkurjev's characteristic zero argument [4, Theorem 2.2]. Namely, consider the map of exact sequences of $k$-group schemes:

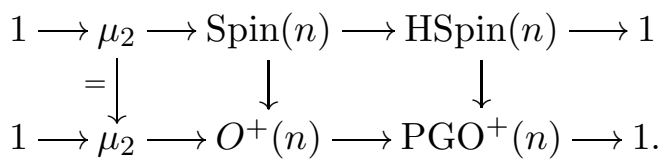

Since HSpin $(n)$ acts generically freely on a half-spin representation, which has dimension $2^{(n-2) / 2}$, we have ed(HSpin $\left.(n)\right) \leq 2^{(n-2) / 2}-n(n-1) / 2$.

By Chernousov-Merkurjev or independently Lötscher, for any normal subgroup scheme $C$ of an affine group scheme $G$ over a field $k$,

$$
\operatorname{ed}(G) \leq \operatorname{ed}(G / C)+\max \operatorname{ed}[E / G],
$$

where the maximum runs over all field extensions $F$ of $k$ and all $G / C$-torsors $E$ over $F$ [4, Proposition 2.1], [14, Example 3.4]. Thus $[E / G]$ is a gerbe over $F$ banded by $C$.

Identifying $H^{2}\left(K, \mu_{p}\right)$ with the $p$-torsion in the Brauer group of $K$, we can talk about the index of an element of $H^{2}\left(K, \mu_{p}\right)$, meaning the degree of the corresponding division algebra over $K$. For a prime number $p$ and a nonzero element $E$ of $H^{2}\left(K, \mu_{p}\right)$ over a field $K$, the essential dimension (or also the $p$-essential dimension) of the corresponding $\mu_{p}$-gerbe over $K$ is equal to the index of $E$, by Karpenko and Merkurjev [11, Theorems 2.1 and 3.1].

By the diagram above, for any field $F$ over $k$, the image of the connecting map

$$
H^{1}(F, \operatorname{HSpin}(n)) \rightarrow H^{2}\left(F, \mu_{2}\right) \subset \operatorname{Br}(F)
$$

is contained in the image of the other connecting map

$$
H^{1}\left(F, P G O^{+}(n)\right) \rightarrow H^{2}\left(F, \mu_{2}\right) \subset \operatorname{Br}(F) .
$$

In the terminology of the Book of Involutions, the image of the latter map consists of the classes $[A]$ of all central simple $F$-algebras $A$ of degree $n$ with a quadratic pair $(\sigma, f)$ of trivial discriminant [12, section 29.F]. Any torsor for $\mathrm{PGO}^{+}(n)$ is split by a field extension of degree a power of 2 , by reducing to the corresponding fact about quadratic forms. So ind $(A)$ must be a power of 2 , but it also divides $n$, and so $\operatorname{ind}(A) \leq 2^{m}$, where $2^{m}$ is the largest power of 2 dividing $n$. We conclude that

$$
\begin{aligned}
\operatorname{ed}(\operatorname{Spin}(n)) & \leq \operatorname{ed}(\operatorname{HSpin}(n))+2^{m} \\
& \leq 2^{(n-2) / 2}-n(n-1) / 2+2^{m} .
\end{aligned}
$$

This completes the proof of the upper bound in Theorem 2.1.

We now prove the corresponding lower bound for the 2-essential dimension of

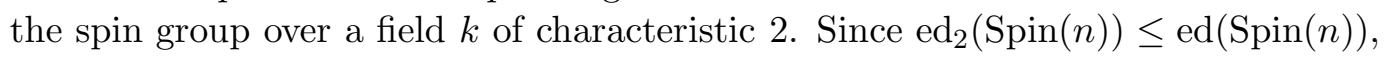


this will imply that the 2-essential dimension and the essential dimension are both equal to the number given in Theorem 2.1.

Write $O(2 r)$ for the orthogonal group of the quadratic form $x_{1} x_{2}+x_{3} x_{4}+\cdots+$ $x_{2 r-1} x_{2 r}$ over $k$, and $O(2 r+1)$ for the orthogonal group of $x_{1} x_{2}+x_{3} x_{4}+\cdots+$ $x_{2 r-1} x_{2 r}+x_{2 r+1}^{2}$. Then we have an inclusion $O(2 r) \subset O(2 r+1)$. Note that $O(2 r)$ is smooth over $k$, with $O(2 r) / O^{+}(2 r) \cong \mathbf{Z} / 2$. The group scheme $O(2 r+1)$ is not smooth over $k$, but it contains a smooth connected subgroup $O^{+}(2 r+1)$ with $O(2 r+1) \cong O^{+}(2 r+1) \times \mu_{2}$. It follows that $O(2 r)$ is contained in $O^{+}(2 r+1)$. Using the subgroup $\mathbf{Z} / 2 \times \mu_{2}$ of $O(2)$, we have a $k$-subgroup scheme $K:=\left(\mathbf{Z} / 2 \times \mu_{2}\right)^{r} \subset$ $O(2 r) \subset O^{+}(2 r+1)$. Let $G$ be the inverse image of $K$ in the double cover $\operatorname{Spin}(2 r+1)$ of $O^{+}(2 r+1)$. Thus $G$ is a central extension

$$
1 \rightarrow \mu_{2} \rightarrow G \rightarrow(\mathbf{Z} / 2)^{r} \times\left(\mu_{2}\right)^{r} \rightarrow 1 .
$$

(Essentially the same "finite Heisenberg group scheme" appeared in the work of Mumford and Sekiguchi on abelian varieties [19, Appendix A].)

To describe the structure of $G$ in more detail, think of $K=\left(\mu_{2}\right)^{r}$ as the 2torsion subgroup scheme of a fixed maximal torus $T_{S O} \cong\left(G_{m}\right)^{r}$ in $O^{+}(2 r+1)$. The chararacter group of $T_{S O}$ is the free abelian group $\mathbf{Z}\left\{x_{1}, \ldots, x_{r}\right\}$, and the Weyl group $W=N\left(T_{S O}\right) / T_{S O}$ of $O^{+}(2 r+1)$ is the semidirect product $S_{r} \ltimes(\mathbf{Z} / 2)^{r}$. Here $S_{r}$ permutes the characters $x_{1}, \ldots, x_{r}$ of $T_{S O}$, and the subgroup $E_{r}=(\mathbf{Z} / 2)^{r}$ of $W$, with generators $\epsilon_{1}, \ldots, \epsilon_{r}$, acts by: $\epsilon_{i}$ changes the sign of $x_{i}$ and fixes $x_{j}$ for $j \neq i$. The character group of $K=T_{S O}[2]$ is $\mathbf{Z} / 2\left\{x_{1}, \ldots, x_{r}\right\}$. The group $E_{r}$ centralizes $K$, and the group $(\mathbf{Z} / 2)^{r} \times\left(\mu_{2}\right)^{r} \subset O^{+}(2 r+1)$ above is $E_{r} \times K$.

Let $L$ be the inverse image of $K$ in $\operatorname{Spin}(2 r+1)$, which is contained in a maximal torus $T$ of $\operatorname{Spin}(2 r+1)$, the inverse image of $T_{S O}$. The character group $X^{*}(T)$ is

$$
\mathbf{Z}\left\{x_{1}, \ldots, x_{r}, A\right\} /\left(2 A=x_{1}+\cdots+x_{r}\right) .
$$

Therefore, the character group $X^{*}(L)$ is

$$
\mathbf{Z}\left\{x_{1}, \ldots, x_{r}, A\right\} /\left(2 x_{i}=0,2 A=x_{1}+\cdots+x_{r}\right) .
$$

(Thus $X^{*}(L)$ is isomorphic to $(\mathbf{Z} / 4) \times(\mathbf{Z} / 2)^{r-1}$, and so $L$ is isomorphic to $\mu_{4} \times$ $\left(\mu_{2}\right)^{r-1}$.) The Weyl group $W$ of $\operatorname{Spin}(2 r+1)$ is the same as that of $O^{+}(2 r+1)$, namely $S_{r} \ltimes E_{r}$. In particular, the element $\epsilon_{i}$ of $E_{r}$ acts on $X^{*}(T)$ by changing the sign of $x_{i}$ and fixing $x_{j}$ for $j \neq i$, and hence it sends $A$ to $A-x_{i}$.

The subset $S$ of $X^{*}(L)$ corresponding to characters of $L$ which are faithful on the center $\mu_{2}$ of $L$ is the complement of the subgroup $X^{*}(K)=\mathbf{Z} / 2\left\{x_{1}, \ldots, x_{r}\right\}$. Therefore, $S$ has order $2^{r}$. The group $E_{r}=(\mathbf{Z} / 2)^{r}$ acts freely and transitively on $S$, since

$$
\left(\prod_{i \in I} \epsilon_{i}\right)(A)=A-\sum_{i \in I} x_{i}
$$

for any subset $I$ of $\{1, \ldots, r\}$.

The group $G=E_{r} \cdot L$ is the central extension considered above. Now, let $V$ be a representation of $G$ over $k$ on which the center $\mu_{2} \subset L$ acts faithfully by scalars. Then the restriction of $V$ to $L$ is fixed (up to isomorphism) by the action of $E_{r}$ on $X^{*}(L)$. By the previous paragraph, the $2^{r}$ 1-dimensional representations of $L$ that are nontrivial on the center $\mu_{2}$ all occur with the same multiplicity in $V$. 
Therefore, $V$ has dimension a multiple of $2^{r}$. This bound is optimal, since the spin representation $W$ of $\operatorname{Spin}(2 r+1)$ has dimension $2^{r}$ over $k$, and the center $\mu_{2}$ acts faithfully by scalars on $W$.

We use the following result of Merkurjev's [15, Theorem 5.2], [11, Remark 4.5]. (The first reference covers the case of the group scheme $\mu_{p}$ in characteristic $p$, as needed here.)

Theorem 2.2. Let $k$ be a field and $p$ be a prime number. Let $1 \rightarrow \mu_{p} \rightarrow G \rightarrow Q \rightarrow 1$ be a central extension of affine group schemes over $k$. For a field extension $K$ of $k$, let $\partial_{K}: H^{1}(K, Q) \rightarrow H^{2}\left(K, \mu_{p}\right)$ be the boundary homomorphism in fppf cohomology. Then the maximal value of the index of $\partial_{K}(E)$, as $K$ ranges over all field extensions of $k$ and $E$ ranges over all $Q$-torsors over $K$, is equal to the greatest common divisor of the dimensions of all representations of $G$ on which $\mu_{p}$ acts by its standard representation.

As mentioned above, for a prime number $p$ and a nonzero element $E$ of $H^{2}\left(K, \mu_{p}\right)$ over a field $K$, the essential dimension (or also the $p$-essential dimension) of the corresponding $\mu_{p}$-gerbe over $K$ is equal to the index of $E$.

Finally, consider a central extension $1 \rightarrow \mu_{p} \rightarrow G \rightarrow Q \rightarrow 1$ of finite group schemes over a field $k$. Generalizing an argument of Brosnan-Reichstein-Vistoli, Karpenko and Merkurjev showed that the $p$-essential dimension of $G$ (and hence the essential dimension of $G$ ) is at least the $p$-essential dimension of the $\mu_{p}$-gerbe over $K$ associated to any $Q$-torsor over any field $K / k$ [11, Theorem 4.2]. By the analysis above of representations of the finite subgroup scheme $G$ of $\operatorname{Spin}(2 r+1)$ over a field $k$ of characteristic 2 , we find that $\operatorname{ed}_{2}(G) \geq 2^{r}$. For a closed subgroup scheme $G$ of a group scheme $L$ over a field $k$ and any prime number $p$, we have $\operatorname{ed}_{p}(L)+\operatorname{dim}(L) \geq$ $\operatorname{ed}_{p}(G)+\operatorname{dim}(G)$ [16, Corollary 4.3] (which covers the case of fppf torsors for nonsmooth group schemes, as needed here). Applying this to the subgroup scheme $G$ of $\operatorname{Spin}(2 r)$, we conclude that $\operatorname{ed}_{2}(\operatorname{Spin}(2 r+1)) \geq 2^{r}-\operatorname{dim}(\operatorname{Spin}(2 r+1))=2^{r}-r(2 r+1)$. Combining this with the upper bound discussed above, we have

$$
\operatorname{ed}(\operatorname{Spin}(2 r+1))=\operatorname{ed}_{2}(\operatorname{Spin}(2 r+1))=2^{r}-r(2 r+1)
$$

for $r \geq 7$.

The proof of the lower bound for $\operatorname{ed}_{2}(\operatorname{Spin}(2 r))$ when $r$ is odd is similar. The intersection of the subgroup $K=\left(\mu_{2} \times \mathbf{Z} / 2\right)^{r} \subset O(2 r)$ with $O^{+}(2 r)$ is $K_{1} \cong$ $\left(\mu_{2}\right)^{r} \times(\mathbf{Z} / 2)^{r-1}$, where $(\mathbf{Z} / 2)^{r-1}$ denotes the kernel of the sum $(\mathbf{Z} / 2)^{r} \rightarrow \mathbf{Z} / 2$. As a result, the double cover $\operatorname{Spin}(2 r)$ contains a subgroup $G_{1}$ which is a central extension

$$
1 \rightarrow \mu_{2} \rightarrow G_{1} \rightarrow(\mathbf{Z} / 2)^{r-1} \times\left(\mu_{2}\right)^{r} \rightarrow 1 .
$$

In this case, an argument analogous to the one for $G$ shows that every representation of $G_{1}$ on which the center $\mu_{2}$ acts by its standard representation has dimension a multiple of $2^{r-1}$ (rather than $2^{r}$ ). The argument is otherwise identical to the $\operatorname{argument}$ for $\operatorname{Spin}(2 r+1)$, and we find that $\operatorname{ed}_{2}(\operatorname{Spin}(2 r)) \geq 2^{r-1}-r(2 r-1)$. For $r$ odd at least 9 , this agrees with the lower bound found earlier, which proves the theorem on $\operatorname{Spin}(n)$ for $n \equiv 0(\bmod 4)$.

It remains to show that for $n$ a multiple of 4 , with $2^{m}$ the largest power of 2 dividing $n$, we have

$$
\operatorname{ed}_{2}(\operatorname{Spin}(n)) \geq 2^{(n-2) / 2}+2^{m}-n(n-1) / 2 .
$$


The argument follows that of Merkurjev in characteristic not 2 [16, Theorem 4.9].

Namely, for $n$ a multiple of 4 , the center $C$ of $G:=\operatorname{Spin}(n)$ is isomorphic to $\mu_{2} \times \mu_{2}$, and $H:=G / C$ is the group $P G O^{+}(n)$. An $H$-torsor over a field $L$ over $k$ is equivalent to a central simple algebra $A$ of degree $n$ over $L$ with a quadratic pair $(\sigma, f)$ and with trivialized discriminant, meaning an isomorphism from the center of the Clifford algebra $C(A, \sigma, f)$ to $L \times L$ [12, section 29.F]. The image of the homomorphism from $C^{*} \cong(\mathbf{Z} / 2)^{2}$ to the Brauer group of $L$ is equal to $\left\{0,[A],\left[C^{+}\right],\left[C^{-}\right]\right\}$, where $C^{+}$and $C^{-}$are the simple components of the Clifford algebra; each is a central simple algebra of degree $2^{(n-2) / 2}$ over $L$. By Merkurjev, there is a field $L$ over $k$ and an $H$-torsor $E$ over $L$ such that ind $\left(C^{+}\right)=\operatorname{ind}\left(C^{-}\right)=$ $2^{(n-2) / 2}$ and $\operatorname{ind}(A)=2^{m}$ [15, section 4.4 and Theorem 5.2]. We use the following result [16, Example 3.7]:

Lemma 2.3. Let $L$ be a field, $p$ a prime number, and $r$ a natural number. Let $C$ be the group scheme $\left(\mu_{p}\right)^{r}$, and let $Y$ be a $C$-gerbe over $L$. Then the p-essential dimension of $Y$, and also the essential dimension of $Y$, is the minimum, over all bases $u_{1}, \ldots, u_{r}$ for $C^{*}$, of $\sum_{i=1}^{r} \operatorname{ind}\left(u_{i}(Y)\right)$.

It follows that the 2 -essential dimension of the $\left(\mu_{2}\right)^{2}$-gerbe $E / G$ over $L$ associated to the $H$-torsor $E$ above is

$$
\operatorname{ed}_{2}(E / G)=\operatorname{ind}(A)+\operatorname{ind}\left(C^{+}\right)=2^{(n-2) / 2}+2^{m} .
$$

It follows that

$$
\begin{aligned}
\operatorname{ed}(\operatorname{Spin}(n)) & \geq \operatorname{ed}_{2}(\operatorname{Spin}(n)) \\
& \geq \operatorname{ed}_{2}(E / G)-\operatorname{dim}(G / C) \\
& =2^{(n-2) / 2}+2^{m}-n(n-1) / 2 .
\end{aligned}
$$

\section{Low-dimensional spin groups}

Rost and Garibaldi determined the essential dimension of the spin groups $\operatorname{Spin}(n)$ with $n \leq 14$ in characteristic not 2 [6, Table 23B]. It should be possible to compute the essential dimension of low-dimensional spin groups in characteristic 2 as well. The following section carries this out for $\operatorname{Spin}(n)$ with $n \leq 10$. We find that in this range (as for $n \geq 15$ ), the essential dimension of the spin group is the same in characteristic 2 as in characteristic not 2, unlike what happens for $O(n)$ and $S O(n)$.

For $n \leq 10$, we give group-theoretic proofs which work almost the same way in any characteristic, despite the distinctive features of quadratic forms in characteristic 2 .

Theorem 3.1. For $n \leq 10$, the essential dimension, as well as the 2-essential dimension, of the split group $\operatorname{Spin}(n)$ over a field $k$ of any characteristic is given 
by:

$\begin{array}{rr}n & \operatorname{ed}(\operatorname{Spin}(n)) \\ \leq 6 & 0 \\ 7 & 4 \\ 8 & 5 \\ 9 & 5 \\ 10 & 4\end{array}$

Proof. As discussed above, it suffices to consider the case of a field $k$ of characteristic 2. For $n \leq 6$, every $\operatorname{Spin}(n)$-torsor over a field is trivial, for example by the exceptional isomorphisms $\operatorname{Spin}(3) \cong S L(2), \operatorname{Spin}(4) \cong S L(2) \times S L(2), \operatorname{Spin}(5) \cong S p(4)$, and $\operatorname{Spin}(6) \cong S L(4)$. It follows that $\operatorname{ed}(\operatorname{Spin}(n))=0$ for $n \leq 6$.

We first recall some general definitions. For a field $k$ of characteristic $p>0$, let $H^{i, j}(k)$ be the etale motivic cohomology group $H_{\mathrm{et}}^{i}(k, \mathbf{Z} / p(j))$, or equivalently

$$
H_{\mathrm{et}}^{i}(k, \mathbf{Z} / p(j)) \cong H_{\mathrm{et}}^{i-j}\left(k, \Omega_{\log }^{j}\right),
$$

where $\Omega_{\log }^{j}$ is the subgroup of the group $\Omega^{j}$ of differential forms on the separable closure $k_{s}$ over $\mathbf{F}_{p}$ spanned by products $\left(d a_{1} / a_{1}\right) \wedge \cdots \wedge\left(d a_{j} / a_{j}\right)$ with $a_{1}, \ldots, a_{j} \in k_{s}^{*}$ [9]. The group $H^{i, j}(k)$ is zero except when $i$ equals $j$ or $j+1$, because $k$ has $p$ cohomological dimension at most 1 [20, section II.2.2]. The symbol $\left\{a_{1}, \ldots, a_{n-1}, b\right]$ denotes the element of $H^{n, n-1}(k)$ which is the product of the elements $a_{i} \in k^{*} /\left(k^{*}\right)^{p} \cong$ $H^{1,1}(k)$ and $b \in k /\left\{a^{p}-a: a \in k\right\} \cong H^{1,0}(k)$.

Also, for a field $k$ of characteristic 2, let $W(k)$ denote the Witt ring of symmetric bilinear forms over $k$, and let $I_{q}(k)$ be the Witt group of nondegenerate quadratic forms over $k$. (By the conventions in section 1, $I_{q}(k)$ consists only of even-dimensional forms.) Then $I_{q}(k)$ is a module over $W(k)$ via tensor product 5 , Lemma 8.16]. Let $I$ be the kernel of the homomorphism rank: $W(k) \rightarrow \mathbf{Z} / 2$, and let

$$
I_{q}^{m}(k)=I^{m-1} \cdot I_{q}(k),
$$

following [5, p. 53]. To motivate the notation, observe that the class of an $m$ fold quadratic Pfister form $\left\langle\left\langle a_{1}, \ldots, a_{m-1}, b\right]\right]$ lies in $I_{q}^{m}(k)$. By definition, for $a_{1}, \ldots, a_{m-1}$ in $k^{*}$ and $b$ in $k,\left\langle\left\langle a_{1}, \ldots, a_{m-1}, b\right]\right]$ is the quadratic form $\left\langle\left\langle a_{1}\right\rangle\right\rangle_{b} \otimes$ $\cdots \otimes\left\langle\left\langle a_{m-1}\right\rangle\right\rangle_{b} \otimes\langle\langle b]]$ of dimension $2^{m}$, where $\langle\langle a\rangle\rangle_{b}$ is the bilinear form $\langle 1, a\rangle$ and $\langle\langle b]]$ is the quadratic form $[1, b]=x^{2}+x y+b y^{2}$.

In analogy with the Milnor conjecture, Kato proved the isomorphism

$$
I_{q}^{m}(F) / I_{q}^{m+1} \cong H^{m, m-1}(F)
$$

for every field $F$ of characteristic 2 [5, Fact 16.2]. The isomorphism takes the quadratic Pfister form $\left\langle\left\langle a_{1}, \ldots, a_{m-1}, b\right]\right\}$ to the symbol $\left\{a_{1}, \ldots, a_{m-1}, b\right]$. (For this paper, it would suffice to have Kato's homomorphism, without knowing that it is an isomorphism.)

We will use the following standard approach to bounding the essential dimension of a group.

Lemma 3.2. Let $G$ be an affine group scheme of finite type over a field $k$. Suppose that $G$ acts on a $k$-scheme $Y$ with a nonempty open orbit $U$. Suppose that for every $G$-torsor $E$ over an infinite field $F$ over $k$, the twisted form $(E \times Y) / G$ of $Y$ over 


$\begin{array}{ccc}n & \operatorname{char} k \neq 2 & \text { char } k=2 \\ 6 & S L(3) \cdot\left(G_{a}\right)^{3} & \text { same } \\ 7 & G_{2} & \text { same } \\ 8 & \operatorname{Spin}(7) & \text { same } \\ 9 & \operatorname{Spin}(7) & \text { same } \\ 10 & \operatorname{Spin}(7) \cdot\left(G_{a}\right)^{8} & \text { same } \\ 11 & S L(5) & \mathbf{Z} / 2 \ltimes S L(5) \\ 12 & S L(6) & \mathbf{Z} / 2 \ltimes S L(6) \\ 13 & S L(3) \times S L(3) & \mathbf{Z} / 2 \ltimes(S L(3) \times S L(3)) \\ 14 & G_{2} \times G_{2} & \mathbf{Z} / 2 \ltimes\left(G_{2} \times G_{2}\right)\end{array}$

Table 1: Generic stabilizer of spin (or half-spin) representation of $\operatorname{Spin}(n)$

$F$ has a Zariski-dense set of F-points. Finally, suppose that $U$ has a $k$-point $x$, and let $N$ be the stabilizer $k$-group scheme of $x$ in $G$. Then

$$
H^{1}(F, N) \rightarrow H^{1}(F, G)
$$

is surjective for every infinite field $F$ over $k$ (or for every field $F$ over $k$, if $G$ is smooth and connected). As a result, $\operatorname{ed}_{k}(G) \leq \operatorname{ed}_{k}(N)$.

The proof is short, the same as that of [6, Theorem 9.3]. (Note that even if $k$ is finite, we get the stated upper bound for the essential dimension of $G$ : a $G$-torsor over a finite field $F$ that contains $k$ causes no problem, because $F$ has transcendence degree 0 over $k$.) If $G$ is smooth and connected, then $H^{1}(F, G)$ is in fact trivial for every finite field $F$ that contains $k$, by Lang [13]; that implies the statement in the theorem that $H^{1}(F, N) \rightarrow H^{1}(F, G)$ is surjective for every field $F$ over $k$.

The assumption about a Zariski-dense set of rational points holds, for example, if $Y$ is a linear representation $V$ of $G$, or if $Y$ is the associated projective space $P(V)$ to a representation, or (as we use later) a product $P(V) \times P(W)$.

We use Garibaldi and Guralnick's calculation of the stabilizer group scheme of a general $k$-point in the spin (for $n$ odd) or a half-spin (for $n$ even) representation $W$ of the split group $\operatorname{Spin}(n)$, listed in Table 1 here [7, Table 1]. Here $\operatorname{Spin}(n)$ has an open orbit on the projective space $P(W)$ of lines in $W$ if $n \leq 12$ or $n=14$, and an open orbit on $W$ if $n=10$. (To be precise, we will use that even if $k$ is finite, there is a $k$-point in the open orbit for which the stabilizer $k$-group scheme is the split group listed in the table.)

We now begin to compute the essential dimension of the split group $G=\operatorname{Spin}(7)$ over a field $k$ of characteristic 2 . Let $W$ be the 8 -dimensional spin representation of $G$. Then $G$ has an open orbit on the projective space $P(W)$ of lines in $W$. By Table 1 , there is a $k$-point $x$ in $W$ whose image in $P(W)$ is in the open orbit such that the stabilizer of $x$ in $G$ is the split exceptional group $G_{2}$. Since $G$ preserves a quadratic form on $W$, the stabilizer $H$ of the corresponding $k$-point in $P(W)$ is at most $G_{2} \times \mu_{2}$. In fact, $H$ is equal to $G_{2} \times \mu_{2}$, because the center $\mu_{2}$ of $G$ acts trivially on $P(W)$.

By Lemma 3.2, the inclusion $G_{2} \times \mu_{2} \hookrightarrow G$ induces a surjection

$$
H^{1}\left(F, G_{2} \times \mu_{2}\right) \rightarrow H^{1}(F, G)
$$


for every field $F$ over $k$. Over any field $F, G_{2}$-torsors up to isomorphism can be identified with 3 -fold quadratic Pfister forms $\left\langle\left\langle a_{1}, a_{2}, b\right]\right.$ ] (with $a_{1}, a_{2} \in F^{*}$ and $b \in F)$, and so $G_{2}$ has essential dimension 3 [20. Théorème 11]. Since $\mu_{2}$ has essential dimension 1, the surjectivity above implies that $G=\operatorname{Spin}(7)$ has essential dimension at most 4 .

Next, a $G$-torsor determines two quadratic forms of dimension 8. Besides the obvious homomorphism $\chi_{1}: G \hookrightarrow \operatorname{Spin}(8) \rightarrow S O(8)$ (which is trivial on the center $\mu_{2}$ of $G$ ), we have the spin representation $\chi_{2}: G \rightarrow S O(8)$, on which $\mu_{2}$ acts faithfully by scalars. Thus a $G$-torsor $u$ over a field $F$ over $k$ determines two quadratic forms of dimension 8 over $F$, which we call $q_{1}$ and $q_{2}$.

To describe these quadratic forms in more detail, use that every $G$-torsor comes from a torsor for $G_{2} \times \mu_{2}$. The two homomorphisms $G_{2} \hookrightarrow G \rightarrow S O(8)$ (via $\chi_{1}$ and $\chi_{2}$ ) are both conjugate to the standard inclusion. Also, $\chi_{1}$ is trivial on the $\mu_{2}$ factor, while $\chi_{2}$ acts faithfully by scalars on the $\mu_{2}$ factor. It follows that $q_{1}$ is a quadratic Pfister form, $\left\langle\langle a, b, c]\right.$ ] (the form associated to a $G_{2}$-torsor), while $q_{2}$ is a scalar multiple of that form, $d\langle\langle a, b, c]]$.

Therefore, a $G$-torsor $u$ canonically determines a 4 -fold quadratic Pfister form,

$$
q_{1}+q_{2}=\langle\langle d, a, b, c]] .
$$

Define $f_{4}(u)$ to be the associated element of $H^{4,3}(F)$,

$$
f_{4}(u)=\{d, a, b, c] .
$$

By construction, this is well-defined and an invariant of $u$. This invariant is normalized (zero on the trivial $G$-torsor) and not zero. (By considering the subgroup $G_{2} \times \mu_{2} \subset \operatorname{Spin}(7)$, where there is a $G_{2} \times \mu_{2}$-torsor associated to any elements $a, b, d$ in $F^{*}$ and $c$ in $F$, we see that $a, b, c, d$ can be chosen arbitrarily. By taking $F$ to be the rational function field $k(a, b, c, d)$, we see that the element $f_{4}(u)=\{d, a, b, c]$ of $H^{4,3}(F)$ can be nonzero. For that, one can use the computation of $H^{n, n-1}$ of a rational function field by Izhboldin [10].)

Therefore, $G=\operatorname{Spin}(7)$ has essential dimension at least 4 . The opposite inequality was proved above, and so $\operatorname{Spin}(7)$ has essential dimension equal to 4 . Since the lower bound is proved by constructing a mod 2 cohomological invariant, this argument also shows that $\operatorname{Spin}(7)$ has 2-essential dimension equal to 4 . For the same reason, the computations of essential dimension below (for $\operatorname{Spin}(n)$ with $8 \leq n \leq 10$ ) also give the 2-essential dimension.

Next, we turn to $\operatorname{Spin}(8)$. At first, let $G=\operatorname{Spin}(2 r)$ for a positive integer $r$ over a field $k$ of characteristic 2 . Let $V$ be the standard $2 r$-dimensional representation of $G$. Then $G$ has an open orbit in the projective space $P(V)$ of lines in $V$. The stabilizer $k$-group scheme $H$ of a general $k$-point in $P(V)$ is conjugate to $\operatorname{Spin}(2 r-1) \cdot Z$, where $Z$ is the center of $\operatorname{Spin}(2 r)$, with $\operatorname{Spin}(2 r-1) \cap Z=\mu_{2}$. (In more detail, a general line in $V$ is spanned by a vector $x$ with $q(x) \neq 0$, where $q$ is the quadratic form on $V$. Then the stabilizer of $x$ in $S O(V)$ is isomorphic to $S O(S)$, where $S:=x^{\perp}$ is a hyperplane in $V$ on which $q$ restricts to a nonsingular quadratic form of dimension $2 r-1$, with $S^{\perp}$ equal to the line $k \cdot x \subset S$.) Here

$$
Z \cong \begin{cases}\mu_{2} \times \mu_{2} & \text { if } r \text { is even } \\ \mu_{4} & \text { if } r \text { is odd }\end{cases}
$$


In particular, if $r$ is even, then $H \cong \operatorname{Spin}(2 r-1) \times \mu_{2}$. Thus, for $r$ even, the inclusion $\operatorname{Spin}(2 r-1) \times \mu_{2} \hookrightarrow G$ induces a surjection

$$
H^{1}\left(F, \operatorname{Spin}(2 r-1) \times \mu_{2}\right) \rightarrow H^{1}(F, G)
$$

for every field $F$ over $k$, by Lemma 3.2 .

It follows that, for $r$ even, the essential dimension of $\operatorname{Spin}(2 r)$ is at most 1 plus the essential dimension of $\operatorname{Spin}(2 r-1)$. Since $\operatorname{Spin}(7)$ has essential dimension 4 , $G=\operatorname{Spin}(8)$ has essential dimension at most 5 .

Before proving that equality holds, let us analyze $G$-torsors in more detail. We know that $H^{1}\left(F, \operatorname{Spin}(7) \times \mu_{2}\right) \rightarrow H^{1}(F, G)$ is onto, for all fields $F$ over $k$. Also, we showed earlier that $H^{1}\left(F, G_{2} \times \mu_{2}\right) \rightarrow H^{1}(F, \operatorname{Spin}(7))$ is surjective. Therefore,

$$
H^{1}\left(F, G_{2} \times \mu_{2} \times \mu_{2}\right) \rightarrow H^{1}(F, G)
$$

is surjective for all fields $F$ over $k$, where $Z=\mu_{2} \times \mu_{2}$ is the center of $G$. As discussed earlier, $G_{2}$-torsors up to isomorphism can be identified with 3 -fold quadratic Pfister forms. It follows that every $G$-torsor is associated to some 3 -fold quadratic Pfister form $\langle\langle a, b, c]]$ and some elements $d, e$ in $F^{*}$, which yield elements of $H^{1}\left(F, \mu_{2}\right)=$ $F^{*} /\left(F^{*}\right)^{2}$.

Next, observe that a $G$-torsor determines several quadratic forms. Besides the obvious double covering $\chi_{1}: G \rightarrow S O(8)$, the two half-spin representations of $G$ give two other homomorphisms $\chi_{2}, \chi_{3}: G \rightarrow S O(8)$. (These three homomorphisms can be viewed as the quotients of $G$ by the three $k$-subgroup schemes of order 2 in $Z$. They are permuted by the group $S_{3}$ of "triality" automorphisms of $G$.) Thus a $G$-torsor $u$ over a field $F$ over $k$ determines three quadratic forms of dimension 8 , which we call $q_{1}, q_{2}, q_{3}$.

To describe how these three quadratic forms are related, use that every $G$ torsor comes from a torsor for $G_{2} \times \mu_{2} \times \mu_{2}$. The three homomorphisms $G_{2} \rightarrow G \rightarrow$ $S O(8)$ (via $\chi_{1}, \chi_{2}$, and $\chi_{3}$ ) are all conjugate to the standard inclusion, whereas the three homomorphisms send $\mu_{2} \times \mu_{2}$ to the center $\mu_{2} \subset S O(8)$ by the three possible surjections. It follows that the three quadratic forms can be written as $q_{1}=d\langle\langle a, b, c]], q_{2}=e\langle\langle a, b, c]]$, and $q_{3}=d e\langle\langle a a, b, c]]$.

Note that a scalar multiple of a quadratic Pfister form, $q=d\left\langle\left\langle a_{1}, \ldots, a_{m-1}, b\right]\right]$ (as a quadratic form up to isomorphism), uniquely determines the associated quadratic Pfister form $q_{0}=\left\langle\left\langle a_{1}, \ldots, a_{m-1}, b\right]\right]$ up to isomorphism. (Proof: it suffices to show that if $q$ and $r$ are $m$-fold quadratic Pfister forms over $F$ with $a q \cong r$ for some $a$ in $F^{*}$, then $q \cong r$. Since $r$ takes value 1 , so does $a q$, and so $q$ takes value $a^{-1}$. But then $a^{-1} q \cong q$ by the multiplicativity of quadratic Pfister forms [5, Corollary 9.9]. Therefore, $r \cong a q \cong q$.)

We now define an invariant for $G=\operatorname{Spin}(8)$ over $k$ with values in $H^{5,4}$. Given a $G$-torsor $u$ over a field $F$ over $k$, consider the three associated quadratic forms $q_{1}, q_{2}, q_{3}$ as above. By the previous paragraph, $q_{1}=d\langle\langle a, b, c]]$ determines the quadratic Pfister form $q_{0}=\langle\langle a, b, c]]$. So $u$ determines the 5 -fold quadratic Pfister form

$$
q_{0}+q_{1}+q_{2}+q_{3}=\langle\langle d, e, a, b, c]]
$$

The associated class

$$
f_{5}(u)=\{d, e, a, b, c] \in H^{5,4}(F)
$$


is therefore an invariant of $u$.

The invariant $f_{5}$ is normalized and not 0 , as shown by considering the subgroup $G_{2} \times Z \subset G=\operatorname{Spin}(8)$, where $Z=\mu_{2} \times \mu_{2}$ : there is a $G_{2} \times Z$-torsor associated to any elements $a, b, d, e$ in $F^{*}$ and $c$ in $F$, and $f_{5}$ of the associated $G$-torsor is $\{d, e, a, b, c]$ in $H^{5,4}(F)$. Therefore, $G$ has essential dimension at least 5. Since the opposite inequality was proved above, $G=\operatorname{Spin}(8)$ has essential dimension over $k$ equal to 5 .

Next, let $G=\operatorname{Spin}(9)$ over a field $k$ of characteristic 2 . Let $W$ be the spin representation of $G$, of dimension 16, corresponding to a homomorphism $G \rightarrow$ $S O(16)$. (A reference for the fact that this self-dual representation is orthogonal in characteristic 2, as in other characteristics, is [8, Theorem 9.2.2].) By Table 1, G has an open orbit on the space $P(W)$ of lines in $W$, and the stabilizer in $G$ of a general $k$-point in $W$ is conjugate to $\operatorname{Spin}(7)$. (This is not the standard inclusion of $\operatorname{Spin}(7)$ in $\operatorname{Spin}(9)$, but rather a lift of the spin representation $\chi_{2}: \operatorname{Spin}(7) \rightarrow S O(8)$ to $\operatorname{Spin}(8)$ followed by the standard inclusion $\operatorname{Spin}(8) \hookrightarrow \operatorname{Spin}(9)$. In particular, the image of $\operatorname{Spin}(7)$ does not contain the center $\mu_{2}$ of $G=\operatorname{Spin}(9)$.) Since $G$ preserves a quadratic form on $W$, it follows that the stabilizer in $G$ of a general $k$-point in $P(W)$ is conjugate to $\operatorname{Spin}(7) \times \mu_{2}$, where $\mu_{2}$ is the center of $\operatorname{Spin}(9)$ (which acts faithfully by scalars on $W)$. Therefore, by Lemma 3.2, the inclusion of $\operatorname{Spin}(7) \times \mu_{2}$ in $G=\operatorname{Spin}(9)$ induces a surjection

$$
H^{1}\left(F, \operatorname{Spin}(7) \times \mu_{2}\right) \rightarrow H^{1}(F, G)
$$

for every field $F$ over $k$.

Since $\operatorname{Spin}(7)$ has essential dimension 4 over $k$ as shown above, $G=\operatorname{Spin}(9)$ has essential dimension at most $4+1=5$.

Next, a $G$-torsor determines several quadratic forms. Besides the obvious homomorphism $R: G \hookrightarrow \operatorname{Spin}(10) \rightarrow S O(10)$, we have the spin representation $S: G \rightarrow$ $S O(16)$. Thus a $G$-torsor over a field $F$ over $k$ determines a quadratic form $r$ of dimension 10 and a quadratic form $s$ of dimension 16 .

To describe how these forms are related, use that every $G$-torsor comes from a torsor for the subgroup $\operatorname{Spin}(7) \times \mu_{2}$ described above. The restriction of $R$ to the given subgroup $\operatorname{Spin}(7)$ is the composition of the spin representation $\chi_{2}: \operatorname{Spin}(7) \rightarrow$ $S O(8)$ with the obvious inclusion $S O(8) \hookrightarrow S O(10)$. The restriction of $S$ to the given subgroup $\operatorname{Spin}(7)$ is the direct sum of the standard representation $\chi_{1}: \operatorname{Spin}(7) \rightarrow$ $S O(8)$ and the spin representation $\chi_{2}: \operatorname{Spin}(7) \rightarrow S O(8)$. Finally, $R$ is trivial on the second factor $\mu_{2}$ (the center of $G$ ), whereas $S$ acts faithfully by scalars on $S$.

Now, let $\left(u_{1}, e\right)$ be a $\operatorname{Spin}(7) \times \mu_{2}$-torsor over $k$, where $u_{1}$ is a $\operatorname{Spin}(7)$-torsor and $e$ is in $H^{1}\left(F, \mu_{2}\right)=F^{*} /\left(F^{*}\right)^{2}$, which we lift to an element $e$ of $F^{*}$. By the earlier analysis of the quadratic forms associated to a $\operatorname{Spin}(7)$-torsor, the quadratic form associated to $u_{1}$ via the standard representation $\chi_{1}: \operatorname{Spin}(7) \rightarrow S O(8)$ is a 3 -fold quadratic Pfister form $\langle\langle a, b, c]]$, while the quadratic form associated to $u_{1}$ via the spin representation $\chi_{2}: \operatorname{Spin}(7) \rightarrow S O(8)$ is a multiple of the same form, $d\langle\langle a, b, c]]$.

By the analysis of representations two paragraphs back, it follows that the quadratic form associated to $\left(u_{1}, e\right)$ via the representation $R: G \rightarrow S O(10)$ is $r=H+d\langle\langle a, b, c]]$, where $H$ is the hyperbolic plane. Also, the quadratic form associated to $\left(u_{1}, e\right)$ via the representation $S: G \rightarrow S O(16)$ is $s=e\langle\langle a, b, c]]+\operatorname{de}\langle\langle a, b, c]]$.

Next, $r$ determines the quadratic form $r_{0}=d\langle\langle a, b, c]]$ by Witt cancellation [5, Theorem 8.4], and that in turn determines the quadratic Pfister form $q_{0}=\langle\langle a, b, c]]$ 
as shown above. Therefore, a $G$-torsor $u$ determines the 5 -fold quadratic Pfister form

$$
q_{0}+r_{0}+s=\langle\langle d, e, a, b, c]]
$$

up to isomorphism.

Therefore, defining

$$
f_{5}(u)=\{d, e, a, b, c]
$$

in $H^{5,4}(F)$ yields an invariant of $u$. By our earlier description of Spin(7)-torsors, we can take $a, b, d, e$ to be any elements of $F^{*}$ and $c$ any element of $F$. Therefore, $f_{5}$ is a nonzero normalized invariant of $G$ over $k$ with values in $H^{5,4}$. It follows that $G$ has essential dimension at least 5 . Since the opposite inequality was proved earlier, $G=\operatorname{Spin}(9)$ over $k$ has essential dimension equal to 5 .

Finally, let $G=\operatorname{Spin}(10)$ over a field $k$ of characteristic 2 . Let $V$ be the 10 dimensional standard representation of $G$, corresponding to the double covering $G \rightarrow S O(10)$, and let $W$ be one of the 16-dimensional half-spin representations of $G$, corresponding to a homomorphism $G \rightarrow S L(16)$. (The other half-spin representation of $G$ is the dual $W^{*}$.)

As discussed above for any group $\operatorname{Spin}(2 r), G=\operatorname{Spin}(10)$ has an open orbit on $P(V)$, with generic stabilizer $\operatorname{Spin}(9) \cdot \mu_{4}$. (Here $\mu_{4}$ is the center of $G$, which contains the center $\mu_{2}$ of $\operatorname{Spin}(9)$.) Consider the action of $G$ on $P(V) \times P(W) \cong \mathbf{P}^{9} \times \mathbf{P}^{15}$. As discussed above, $\operatorname{Spin}(9)$ (and hence $\operatorname{Spin}(9) \cdot \mu_{4}$ ) has an open orbit on $P(W)$. As a result, $G$ has an open orbit on $P(V) \times P(W)$. Moreover, the generic stabilizer of $\operatorname{Spin}(9)$ on $P(W)$ is $\operatorname{Spin}(7) \times \mu_{2}$, where the inclusion $\operatorname{Spin}(7) \hookrightarrow \operatorname{Spin}(9)$ is the composition of the spin representation $\operatorname{Spin}(7) \hookrightarrow \operatorname{Spin}(8)$ with the standard inclusion into $\operatorname{Spin}(9)$; in particular, the image does not contain the center $\mu_{2}$ of $\operatorname{Spin}(9)$. Therefore, the generic stabilizer of $\operatorname{Spin}(9) \cdot \mu_{4} \subset \operatorname{Spin}(10)$ on $P(W)$ is $\operatorname{Spin}(7) \times \mu_{4}$. We conclude that $G$ has an open orbit on $P(V) \times P(W)$, with generic stabilizer $\operatorname{Spin}(7) \times \mu_{4}$. It follows that

$$
H^{1}\left(F, \operatorname{Spin}(7) \times \mu_{4}\right) \rightarrow H^{1}(F, G)
$$

is surjective for every field $F$ over $k$, by Lemma 3.2

The image $H_{2}$ of the subgroup $H=\operatorname{Spin}(7) \times \mu_{4} \subset G$ in $S O(10)$ is $\operatorname{Spin}(7) \times \mu_{2}$, where $\operatorname{Spin}(7)$ is contained in $S O(8)$ (and contains the center $\mu_{2}$ of $S O(8)$ ) and $\mu_{2}$ is the center of $S O(10)$. In terms of the subgroup $S O(8) \times S O(2)$ of $S O(10)$, we can also describe $H_{2}$ as $\operatorname{Spin}(7) \times \mu_{2}$, where $\operatorname{Spin}(7)$ is contained in $S O(8)$ and $\mu_{2}$ is contained in $S O(2)$. Thus $H_{2}$ is contained in $\operatorname{Spin}(7) \times S O(2)$. Therefore, $H$ is contained in $\operatorname{Spin}(7) \times G_{m} \subset G=\operatorname{Spin}(10)$, where the multiplicative group $G_{m}$ is the inverse image in $G$ of $S O(2) \subset S O(10)$. It follows that

$$
H^{1}\left(F, \operatorname{Spin}(7) \times G_{m}\right) \rightarrow H^{1}(F, G)
$$

is surjective for every field $F$ over $k$. Since every $G_{m}$-torsor over a field is trivial,

$$
H^{1}(F, \operatorname{Spin}(7)) \rightarrow H^{1}(F, G)
$$

is surjective for every field $F$ over $k$. 
Here $\operatorname{Spin}(7)$ maps into $\operatorname{Spin}(8)$ by the spin representation, and then $\operatorname{Spin}(8) \hookrightarrow$ $G=\operatorname{Spin}(10)$ by the standard inclusion. By the description above of the 8dimensional quadratic form associated to a Spin(7)-torsor by the spin representation, it follows that the quadratic form associated to a $G$-torsor is of the form $H+d\langle\langle a, b, c]]$.

Every 10-dimensional quadratic form in $I_{q}^{3}$ over a field is associated to some $G$-torsor. So we have given another proof that every 10-dimensional quadratic form in $I_{q}^{3}$ is isotropic. This was proved in characteristic not 2 by Pfister, and it was extended to characteristic 2 by Baeza and Tits, independently [2, pp. 129-130], 21. Theorem 4.4.1(ii)].

Since $\operatorname{Spin}(7)$ has essential dimension 4, the surjectivity above implies that $G=$ $\operatorname{Spin}(10)$ has essential dimension at most 4 . To prove equality, we define a nonzero normalized invariant for $G$ with values in $H^{4,3}$ by the same argument used for $\operatorname{Spin}(7)$. Namely, a $G$-torsor $u$ over a field $F$ over $k$ determines a 4 -fold quadratic Pfister form

$$
\langle\langle d, a, b, c]]
$$

up to isomorphism, and hence the element

$$
f_{4}(u)=\{d, a, b, c]
$$

in $H^{4,3}(F)$. This completes the proof that $G=\operatorname{Spin}(10)$ over $k$ has essential dimension equal to 4 . As in the previous cases, since the lower bound is proved using a mod 2 cohomological invariant, $G$ also has 2-essential dimension equal to 4 .

\section{References}

[1] A. Babic and V. Chernousov. Lower bounds for essential dimensions in characteristic 2 via orthogonal representations. Pac. J. Math. 279 (2015), 36-63. 1. 3

[2] R. Baeza. Quadratic forms over semilocal rings. Lecture Notes in Mathematics 655, Springer (1978). 14

[3] P. Brosnan, Z. Reichstein, and A. Vistoli. Essential dimension, spinor groups and quadratic forms. Ann. Math. 171 (2010), 533-544. 1, 2

[4] V. Chernousov and A. Merkurjev. Essential dimension of spinor and Clifford groups. Algebra Number Theory 2 (2014), 457-472. 1, 3, 4.

[5] R. Elman, N. Karpenko, and A. Merkurjev. The algebraic and geometric theory of quadratic forms. Amer. Math. Soc. (2008). 8, 11, 12

[6] S. Garibaldi. Cohomological invariants: exceptional groups and spin groups. Mem. Amer. Math. Soc. 200 (2009), no. 937. 1, 7, 9]

[7] S. Garibaldi and R. Guralnick. Spinors and essential dimension. Compos. Math., to appear. 1, 3, 9

[8] S. Garibaldi and D. Nakano. Bilinear and quadratic forms on rational modules of split reductive groups. Canad. J. Math. 68 (2016), 395-421. 12 
[9] T. Geisser and M. Levine. The $K$-theory of fields in characteristic $p$. Invent. Math. 139 (2000), 459-493. 8

[10] O. Izhboldin. On the cohomology groups of the field of rational functions. Mathematics in St. Petersburg, 21-44, Amer. Math. Soc. Transl. Ser. 2, 174, Amer. Math. Soc. (1996). 10

[11] N. Karpenko and A. Merkurjev. Essential dimension of finite p-groups. Invent. Math. 172 (2008), 491-508. 4, 6

[12] M.-A. Knus, A. Merkurjev, M. Rost, and J.-P. Tignol. The book of involutions. Amer. Math. Soc. (1998). 2, 4, 7]

[13] S. Lang. Algebraic groups over finite fields. Amer. J. Math. 78 (1956), 555-563. 9

[14] R. Lötscher. A fiber dimension theorem for essential and canonical dimension. Compos. Math. 149 (2013), 148-174. 4

[15] A. Merkurjev. Maximal indexes of Tits algebras. Doc. Math. 1 (1996), 229-243. 6. 7

[16] A. Merkurjev. Essential dimension. Quadratic forms - algebra, arithmetic, and geometry, 299-325, Contemp. Math., 493, Amer. Math. Soc. (2009). 1, 6, 7

[17] A. Merkurjev. Essential dimension. Bull. Amer. Math. Soc., to appear. 11, 2

[18] Z. Reichstein. Essential dimension. Proceedings of the International Congress of Mathematicians, v. II, 162-188. Hindustan Book Agency, New Delhi (2010). 1. 2

[19] T. Sekiguchi. On projective normality of abelian varieties. II. J. Math. Soc. Japan 29 (1977), 709-727. 5

[20] J.-P. Serre. Galois cohomology. Springer (2002). 8, 10]

[21] J. Tits. Strongly inner anisotropic forms of simple algebraic groups. J. Algebra 131 (1990), 648-677. 14

UCla Mathematics Department, Box 951555, Los Angeles, CA 90095-1555 TOTARO@MATH.UCLA.EDU 